\title{
Cultivating Academic Integrity in a Digital Learning Environment
}

\author{
Imani Akin, Elizabeth Johnson \\ American College of Education
}

\begin{abstract}
Concerns regarding academic integrity within online or virtual learning environments is prominent. A proactive approach and reminders of the fundamentals of academic integrity allows for intervals of instruction and reflection for scholars. Fostering a culture of academic integrity through an academic community beyond the course and within the institution can support ethical responsibility. The gap in the literature is a lack of a perceptions from online professors and administration and a lack of definition for academic integrity. Academic integrity is defined as what academic integrity is not and focuses on negative aspects. Phenomenology was the research methodology used to examine institutional processes to promote academic integrity within online or virtual programs in higher education with data collected from 20 online instructors, staff and administrators. Research question guiding this study asked what evidence-based procedures and processes dissuade ethical violations. A review of the literature includes an examination of the planned behavior theory to support student and institutional success in promoting academic integrity. Data was collected through SurveyMonkey in an open-ended question format on experiences with institutional policy enforcement and perceptions of academic integrity. Key results include a disconnect in communication perception among virtual institutions. Recommendations for cultivating academic integrity, as well as a definition for academic integrity. Online professors, and administration will benefit from the research to cultivate academic integrity in a digital learning environment.
\end{abstract}

\section{Introduction}

Academic integrity was a long-standing topic in education with an increasing issue in institutional supervision [3]. Technology had changed teaching and learning and created new pathways for learning. Teaching and maintaining academic integrity was critical for all learning environments, including, but not inclusive to face-to face-and virtual learning environments. In the fall of 2014, the United States reported 5.8 million students enrolled in online courses [13]. A review of policy, processes, and procedures for promoting academic integrity addressed newer learning environments.

\section{Academic Integrity in Online Environments}

There was concern of incidents regarding academic dishonesty within online or virtual learning environments. Opportunities for violation of academic integrity in online learning environments was increasing. While there was a concern of incidents regarding academic integrity within online or virtual learning environments there was no deficiency on research on the concept of academic ethics, honor, and plagiarism both online, and face to face learning environment [8].

\section{Purpose of the Study}

Identifying proactive processes to promote academic integrity online supported the development of processes and procedures to actively plan and foster a culture of academic responsibility. Phenomenologically surveying online faculty and staff with open ended questions regarding lived experiences regarding academic integrity issues supported this pilot study. A random selection process using an online survey provided initial insight into the activities surrounding academic integrity. The research question guiding this study was: What evidence-based procedures and processes dissuade ethical violations?

\section{Literature Review}

Promoting academic integrity in a virtual learning environment had similarities to promoting academic integrity to an in-seat setting [14]. There were a few issues which were not present at in-seat institutions. The review of literature included information on planned behavior theory to form a theoretical frame work aligns with the purpose of promoting positive integrity in academic settings. The research included 
studies of academic misconduct and behaviors promoting and preventing academic integrity. Motivation for practicing academic integrity were explored. Ethical issues, effects, and deterrence were addressed along with methods to encourage academic integrity.

\subsection{Defining Academic Integrity}

Developing a global definition for academic integrity may not be possible. Defining academic integrity was challenging due to the absence of a generally accepted definition but could be classified as honest, responsible scholarship [14]. Defining academic dishonesty was easier. A consensus among some higher education faculty and staff was plagiarism or fabrication was a violation of academic integrity. Plagiarism is the utilization of another's intellectual property as new work $[1,8]$.

Self-plagiarism is noted as resubmitting previously submitted work as new and original information [1]. Ghost writing is another form of plagiarism in which students pay another to write portions or entire works with the student noted as the author [2]. Fabrication can either be citing work as an author's which is untrue, or, it can be presenting false information with acknowledgement either given or not given to an author $[1 ; 8]$.

\subsection{Global Incidences of Academic Misconduct and Violations}

Academic misbehavior is noted as a scam, or con, when the misconduct is not overt, but counteractive to expectations, such as falsely stating technical issues, or software problems as reasons for lateness, or willful inaccuracies [11]. Falsification of personal or family illness, personal or family emergency and family or loved one death to extend deadlines is determined to be academic misbehavior [1, 17] Academic fraud extends to impersonation of another person in lieu of a degree, being deceitful with identity or circumstances, or hindering the educational or institutional process through interference of a student or faculty member [3]. Providing examples of academic behaviors leading to academic dishonesty aids in a universal perception of defining academic integrity [4].

The core of the acceptance of academic dishonesty lead to the culture of cheating [16]. The culture of cheating indicated the socially acceptable norms and behaviors in an academic setting $[4,16]$. The students, and not the institutions, directly impacted the acceptability of the cheating culture in online courses $[14,16]$. There was global acceptance of the misnomer academic dishonesty was more prevalent in online courses due to a lack of physical interaction with institutional personnel $[2,3,16]$. The extensive use of unfair technological devices and reliance on internet search engines was a manipulation of valid student academic assessment [17]. The burden of proof for student assessment was the responsibility of institutions [3, 17].

Information from a review of the literature, addresses the challenges of one country due to a series of famous plagiarism cases [5]. Institutions of higher education in one country launched official investigations due to online activists challenging the writings of prominent legislators. Issues surrounded the integrity of the politicians' dissertations and, in several circumstances, the institutions decided to revoke doctoral degrees. The prime minister of the country was also one whose doctoral degree was revoked when the dissertation was found to be plagiarized in some part. The institution and country's academia encountered months of public scrutiny.

\subsection{Academic Integrity for Collaborative Research}

Issues of group academic misconduct should be addressed. Researchers shared experiences of a request to collect data without informed consent by community partners [8]. What should have been a simple resolution to gain consent or discontinue the collaboration was more challenging. Recommendations for building an ethical framework when collaborating with other on research.

\subsection{Avoiding Academic Violations}

In developing self-management abilities of emotional intelligence (awareness, regulation, and motivation) individuals will become extra conscious of personal behavior and emotions and the positive and negative the impact of both, on others [10]. Learning to control disrupting actions, deliberate before acting, and increasing intrinsic motivation, can lead to greater determination during challenging circumstances. individuals can better read and react to others' emotional states and more effectively build relationships and broaden personal and professional networks

\subsection{Theoretical Framework for Promoting Academic Integrity}

A theoretical framework appropriate for managing the issue and the behavior included the theory of planned behavior. Incorporation of the dimension of perceived control with the theory of reasoned action led Icek Aizen to development of the theory of planned behavior. The premise of theory of planned behavior was intention drives behavior. The three dimensions affecting intention included attitude, subjective norms and perceived behavior control. The theory of planned behavior can be a personal model 
used to predict academic misconduct [15]. Integration of components and or concepts of planned behavior theory into the curriculum can aid in promoting academic integrity. Theory of Planned Behavior suggests intention drives behavior.

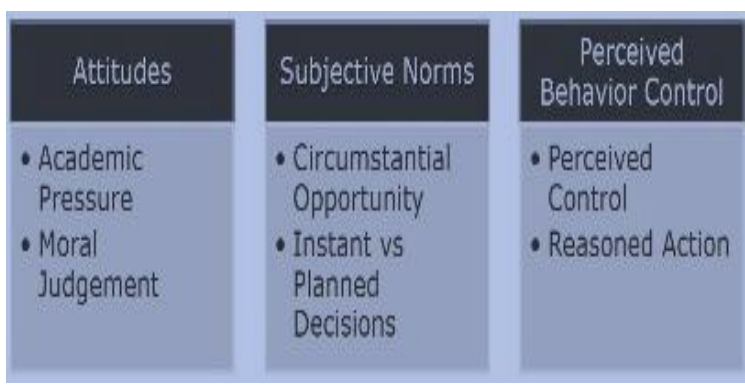

Figure 1. Three Dimensions of the Theory of Planned Behavior

\subsection{Integrating Integrity into the Curriculum}

Student embracing the intrinsic value of doing the right thing was a factor to address. Faculty and staff can affect the subjective norm by incorporating the value of integrity within academics [15]. A positive or negative mood can be redirected by fostering a sense of safety. Faculty and staff can impact perceived behavior control by encouraging positive behavior and a sense of efficacy when integrating multiple successes within the course curriculum planning. The students' perception of the ability to manage the behavior was the goal. Assessing the attitude of students can be addressed through planning for student preparedness and readiness for choosing the positive behavior.

\subsection{Consequences for Academic Dishonesty}

Researchers recommend consideration of the motivation for academic dishonesty prior to determining consequences [7]. A degree awarded by an academic institution serves as evidence an individual has demonstrated satisfactory mastery of the required knowledge and skills, while society receives this testament in good faith [3]. Additional research on proactive processes to dissuade ethical violations and promote academic integrity in academia can contribute to the literature on this issue. Stricter policies and punishments have served as processes and procedures to deter unethical behaviors. Promoting a community or culture, which values the fundamental of academic integrity, will provide students and faculty with a clearer understanding of the "gray areas" which are beyond cheating, and plagiarism [12]. Reminders to deter students from forgetting policies, as well as encouraging professors to uphold set policies by reporting incidents was recommended from previous studies [12].

\section{Method}

The cases of unethical behavior within doctoral programs were of interest as the terminal degree is considered a higher level of academic honor and prestige [8]. The phenomenological pilot study to identify proactive methods for promoting academic integrity included interviewing 10 online instructor and administrators. The research questions were designed to gauge the who, what, where and how of the levels of the responsibility for academic was managed. The seven-question survey was developed in Survey Monkey and disseminated through LinkedIn. The data was retrieved and analyzed.

The study was extended to determine how online professors and administrators defined academic integrity. A total of 12 academic professors and administrators were asked to participate in the openended prompt. The prompt was designed to explore the perceptions of how online professors and administrators perceived academic integrity based on personal and professional experience. The one prompt was developed and disseminated via email. The data was retrieved and analyzed.

\section{Findings}

Research questions 1 and 2 allowed for collection of demographic data. The questions included asking respondents about the position or role in higher education and how many years the respondents worked in higher education. $10 \%$ of the respondents were in administrative positions, $30 \%$ were faculty, and $60 \%$ of the survey was completed by adjunct faculty. Communication of Academic Integrity.

Respondents reported the communication channels and methods used to promote academic integrity. Out of the responses, $40 \%$ indicated email was used to promote academic integrity, $30 \%$ shared the syllabi contained information on academic integrity, the course, and course policies. $20 \%$ of the respondents indicated assessment feedback was used to promote academic integrity, academic integrity was addressed in the handbook, in orientation, and covered in student commons. $10 \%$ of the responses included that information was posted in the library, announcements, and everywhere was included in the response.

\subsection{Communication of Academic Integrity Policy}

In response to who enforces the academic integrity policy the participants provided multiple responses. The academic dean was named in $100 \%$ of the responses. Department and program chairs were identified as enforcers of academic integrity in $70 \%$ of the responses as were faculty. One respondent 
responded all were responsible for enforcing academic integrity while another respondent replied academic integrity policies were enforced; At the end of day by the CAO.... In day-to-day practice, by the Institutional Research Board, by a cadre of Institutional Research Reviewers (IRRs) who review completed dissertations etc. Responses included details of the process from the instructor noticing then reporting the violation using an institution form to forward the matter to administration and another response named a student review committee.

Table 1 . How is the academic integrity policy being communicated throughout the institution, such as student areas, faculty and staff areas and in courses?

\begin{tabular}{c|l}
\hline Frequency & \multicolumn{1}{|c}{ Responses } \\
\hline $40 \%$ & Email communication \\
\hline $30 \%$ & Syllabi \\
\hline $30 \%$ & $\begin{array}{l}\text { Courses and Course } \\
\text { Policies }\end{array}$ \\
\hline $20 \%$ & Assessment Feedback \\
\hline $20 \%$ & Handbook \\
\hline $20 \%$ & Student Orientation \\
\hline $20 \%$ & Student Commons \\
\hline $10 \%$ & $\begin{array}{l}\text { Information posted in } \\
\text { library }\end{array}$ \\
\hline $10 \%$ & Announcements \\
\hline $10 \%$ & Everywhere \\
\hline
\end{tabular}

\subsection{Enforcing Academic Integrity}

In response to who enforces the academic integrity policy the participants provided multiple responses. The academic dean was named in $100 \%$ of the responses. Department and program chairs were identified as enforcers of academic integrity in $70 \%$ of the responses as were faculty. One respondent responded all were responsible for enforcing academic integrity while another respondent replied academic integrity policies were enforced; At the end of day by the CAO.... In day-to-day practice, by the Institutional Research Board, by a cadre of Institutional Research Reviewers (IRRs) who review completed dissertations etc. Responses included details of the process from the instructor noticing then reporting the violation using an institution form to forward the matter to administration and another response named a student review committee.
Table 2. Who enforces the academic integrity policy?

\begin{tabular}{l|l}
\hline Frequency & Responses were multiple \\
\hline $\mathbf{1 0 0} \%$ & Academic Dean \\
\hline $\mathbf{7 0} \%$ & $\begin{array}{l}\text { Department and Program } \\
\text { Chairs, Administrators, } \\
\text { Directors }\end{array}$ \\
\hline $\mathbf{7 0} \%$ & Faculty \\
\hline $\mathbf{1 0} \%$ & $\begin{array}{l}\text { Institutional Review Board, } \\
\text { Institutional Research } \\
\text { Reviewers }\end{array}$ \\
\hline $\mathbf{1 0} \%$ & Everyone \\
\hline
\end{tabular}

\subsection{Supporting Reports of Violations of Academic Integrity}

Table 3. Do you feel supported when you report violations of the academic integrity policy? If so how? If not how?

\begin{tabular}{l} 
Administration \\
Following protocol and faculty need to know the \\
steps \\
Faculty \\
\hline F 1 If we are in agreement then yes. Sometimes the \\
policy is overridden and that can be frustrating. \\
F 2 Yes, the reporting process requires \\
documentation and as long as the documentation \\
is adequate there are no issues proceeding with \\
the policy \\
F 3 I feel supported since the leadership has shown \\
examples of how they support faculty. We are \\
told to keep documentation lively and available in \\
our courses. \\
Adjunct \\
A 1 When I follow procedures, I have no problems \\
A 2 Web-based plagiarism programs are key, \\
at times, \\
A 3 No pushback from administrators, \\
A 4 If we are in agreement, sometimes policy is \\
overridden \\
A 5 I am unsure, I have never been told any results
\end{tabular}

When asked if and how support was provided, $60 \%$ of the respondents felt supported, of those who responded yes, many of them further cited evidence. $30 \%$ of the educators were not sure. One respondent, of the $30 \%$ was unsure and shared the frustration of sometimes the policy being overridden. $10 \%$ did not address the question but mentioned an academic integrity tool as helpful without addressing. 


\subsection{Processes and Procedures}

A total of $40 \%$ of respondents indicated speaking directly with the participant as part of the process for reporting and managing incidents. Another $40 \%$ reported the matter was forwarded to supervisors or administration. An online reporting system was identified by $10 \%$ of participants, while $10 \%$ reported being unsure of the reporting process.

Table 4. What is the process for reporting and managing an incident when you find that a student did not follow academic requirements?

\begin{tabular}{l|l}
\hline Participants & Responses \\
\hline Administration & $\begin{array}{l}\text { Report to admin, administration } \\
\text { investigates, follows up with } \\
\text { faculty, and consequences for } \\
\text { the student }\end{array}$ \\
\hline Faculty & $\begin{array}{l}\text { First a discussion with the } \\
\text { student, then the Academic } \\
\text { Coordinator, } \\
\text { There is an online reporting } \\
\text { system. }\end{array}$ \\
\hline Adjunct & $\begin{array}{l}\text { First a warning to the student, } \\
\text { then 0 for a grade, third } \\
\text { infraction is course failure and } \\
\text { possible expulsion } \\
\text { I am unsure, it is in the syllabus } \\
\text { First address the student, then } \\
\text { forward the issue to the next } \\
\text { level of authority } \\
\text { Contact leads } \\
\text { Email to direct supervisor } \\
\text { 1. Speak with the student 2. } \\
\text { Create proper documentation 3. } \\
\text { Contact the appropriate } \\
\text { administrator 4. Investigate 5. } \\
\text { Administration handles student } \\
\text { consequences }\end{array}$ \\
\hline
\end{tabular}

\subsection{Fidelity in the Promotion of Institutional Academic Integrity}

Within the list of actions $50 \%$ of the respondents indicated communication was needed. Some indicated communication of policies must clearly be set by administrators, another stated communication must be everywhere and clear for students, and another depicted personal integrity and communication with faculty was needed. $20 \%$ of respondents indicated quizzes and embedded tutorials should be implemented in courses. $20 \%$ of respondents indicated the usage of TurnItIn, a web-based, plagiarism prevention service developed by iParadigms, LLC would help monitor and student work. $10 \%$ of respondents indicated a policy of zeros, without resubmission and possible expulsion would be a deterrent and another implied there was nothing applicable for their situation.

Table 5. What ideas or actions do you recommend to ensure fidelity in the promotion of institutional academic integrity?

\begin{tabular}{l}
\hline Administration \\
\hline Quizzes for students and faculty to understand \\
what is and is not plagiarism, understanding of \\
intentional/unintentional plagiarism, activities for \\
faculty and students to demonstrate understanding \\
\hline Faculty \\
F 1 Communicate everywhere so that it is clear for \\
students \\
F 2 The use of TurnItIn in assignments and \\
discussions. \\
F 3 Use customized test question rather than \\
publisher test bank that may be compromised. \\
Zeros, no resubmissions, possible expulsion \\
\hline Adjunct
\end{tabular}

A 1 Policies must be clear with as few interpretations as possible, administration must implement consequences without favoritism, students should sign off they have read the student handbook

A 2 Students need personal integrity and to follow policies, reach out to faculty

A 3 Use web-based programs, students need academic integrity, and how administration monitors this should be part of student and new faculty orientation

A 4 Videos in each course, mandatory tutorials with quizzes

A 5. I believe that increased communication would always enhance the process. Additionally, I believe that instructors should receive some notification regarding the outcome of the investigation.

\subsection{Educators Data of Academic Integrity}

A total of 12 online professors and administrators were asked to participate in an online survey administered via email. Responses were made by seven of the twelve participants. The five participants who did not respond were not included. Online professors and administrators were asked to define academic integrity based on personal and professional experience. The prompt was open ended allowing participants to respond based on perception. No other prompt or question was asked. After the professor's and administrator's definition responses were collected via email, the data was scrubbed to remove personal identification to protect participant confidentiality. 
Table 6. Drawing on personal and professional experiences, please explain your definition of academic integrity.

\begin{tabular}{l} 
Responses \\
P 1 Academic integrity is consistently ensuring \\
original works are submitted as the author's own \\
contributions and proper citations are utilized to \\
credit other's contributions and resources \\
accordingly. \\
P 2 Not intentionally using other people's specific \\
ideas/writing without attribution. \\
P3 Academic integrity is ensuring honesty. A \\
scholar will provide original thought and analysis. \\
Any thoughts, perspectives, or opinions not \\
developed from that scholar would be credited to \\
the author. \\
\hline P4 Being honest about research, protecting research \\
subjects, giving credit for sources cited and experts \\
consulted. Only presenting your UNIQUE ideas as \\
your own. \\
P5 When I think of academic integrity, the first \\
value that comes to my mind is honesty. Most often, \\
a lack of academic integrity is evident when \\
someone presents ideas of others as their own. The \\
International Center for Academic Integrity adds \\
other values to its definition: honesty, trust, \\
fairness, respect, and courage. \\
\hline P6 Upholding ethical standards related to creating \\
original work and thought relevant to the academic \\
field. \\
P7 Being accountable for your original ideas and \\
the ideas of others. When you use other's opinions \\
and ideas you are ensuring that you are giving credit \\
to the original author of the work. In addition, you \\
are clearly identifying your own ideas so that others \\
can share your work in a public manner with \\
honesty.
\end{tabular}

The responses were evaluated for similar and common words or phrases. The word honest was mentioned in $71 \%$ of definitions. Original was a key word in $71 \%$ of explanations, with original work specifically mentioned in $29 \%$ of definitions. Ethical was identified in $14 \%$ of participant verbiage. The word ensuring was noted in $43 \%$ of comments. Phrases such as give credit, or credit the author was listed in $71 \%$ of responses. Contributions and opinions were presented in $29 \%$ of participant explanations individually, while ideas were mentioned in $100 \%$ of definitions from professors and administrators. This indicated key elements of academic integrity should reflect the responses of: honest, original, contributions or ideas or opinions, ensuring, give credit/credit the author and original work are fundamental in the development of the definition of academic integrity.

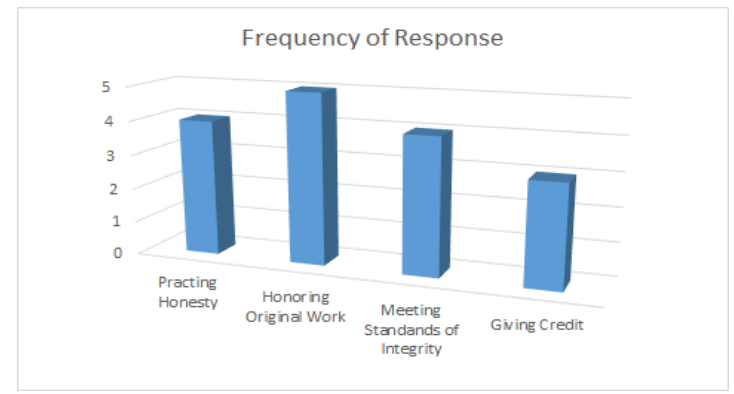

Figure 2. Emerging Themes Regarding Academic Integrity

\section{Application of Theory to Teaching and Learning}

Data from participants and the literature revealed strategies for use of the dimensions of the theory of planned behavior.

Dimension I: Attitudes which are impacted by academic pressure and current moral judgement can be integrated into the curriculum through one-to-one student conferences or added to course policy.

Dimension II: Subjective Norms which are impacted by circumstantial opportunity and instant versus planned behavior can be offset by scaffolding support for students and providing safety nets for student to gain assurance and to avoid punitive consequence.

Dimension III: Perceived Behavior Control triggered by perceived control and reasoned actions can be offset by incorporating structure for students to develop habits of mind through consistent practice and introducing opportunity to study growth mindset theory and see growth mindset in action.

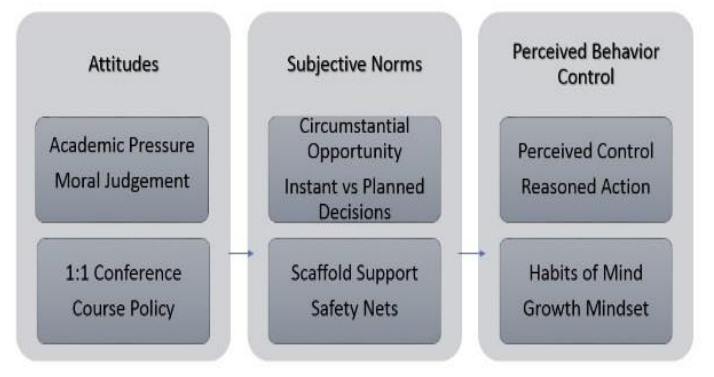

Figure 3. Integrated Components of TPB in Curriculum

\section{Working Definition for Academic Integrity}

Additional research included a working definition of academic indemnity. Practical application of the planned behavior theory includes strategies to support students' habits and perceptions. Strategies include scaffolding and incorporations safety nets to offset the 
needs to participate in an academic violation. Interpretation of findings identified a need for a working definition of academic integrity to narrow the gap in the literature.

Current literature defines academic dishonesty but identifies academic integrity as avoidance of academic dishonesty. A random selection of faculty, staff, and administration from were asked to complete a questionnaire with one open sentence questions. Data was collected and analyzed to develop a comprehensive working definition for academic integrity. From the literature, and from the data collected a working definition was developed.

Academic integrity means practicing honesty by following rules and procedures of the Professional Standards to meet the mission, vision, and goals of the institution, while adhering to the standards of originality in writing by providing authentic scholarship and ensuring credit for the original work of others.

\section{Implications for Institutions}

Implications of the findings indicate the accountability has become blurred. The need for defined roles and responsibilities are important in sustaining and monitoring the academic curriculum, policies, which promote academic integrity. A disconnect between what is known is a loophole for some to manipulate policies. This seemingly leaves honest students at a disadvantage when the dishonest seem to escape consequences. The issue of responsibility and directing one to another office ensures there is a known chasm in student academic integrity instruction no one is willing to address. Institutions cannot rely on student moral compasses to ensure integrity is adhered to by students. The idea everyone is doing all which can be done, but the problem continues is a misnomer. Collectively and holistically an institution is judged on the standards set forth. If accountability and responsibility is blurred sustainability of policies is at risk.

\section{Recommendations for Leadership}

Half of the respondents indicated communication was needed. Some indicated communication of policies must clearly be set by administrators, another stated communication must be everywhere and clear for students, and another depicted personal integrity and communication with faculty was needed. Adjunct professors typically identified policies and procedures with transparency and visibility were key elements. Faculty noted clear communication for students, and listed proactive methods to curtail cheating and plagiarism through instructional tools and plagiarism programs. Faculty named situational consequences for students.
Administrators who responded provided holistic approaches for proactive deterrents. Professional development opportunities for faculty, as well as instruction information for students were suggested to develop a clear understand of the institutional stance.

Recurring themes from the data depict adjunct or new hires are unaware or unfamiliar with policies, or how to deal with situations. A suggestion would be for professional development be a proactive measure to promote academic integrity as colleges are relying more on adjunct professors to work in place of tenured faculty. Considering adjunct professors are in the forefront of working with students it is essential awareness, availability, and clarity regarding academic integrity is prevalent. Concurrently, faculty should be proactive in learning, and adhering to the institutional policies and procedures. Faculty are the ones students look to for instruction and guidance.

Another recurring theme is administration viewed the policies as being in place, and thereby should be known. Administrative responses indicated a need to make issues of academic dishonesty situations less accessible for students, while maintaining adjunct and full-time faculty are following the policy. When evaluating responses from the collective group, it is clear academic integrity policies and procedures are not clear to faculty and staff. Even if the policies and procedures are considered available by administration.

\section{Summary}

The summary of the findings indicated $100 \%$ of the respondents identified the academic dean as the enforcer of academic integrity, but all participants identified an administrator as responsible. Only $10 \%$ of respondents noted all members of faculty and staff are responsible to enforce the academic integrity policies of the institution. Considering the first responders to observe a violation is typically the faculty the question remains as to why many in an academic institution not accept the role to uphold the academic integrity set forth by an institution. Perhaps professors feel bound to follow procedures, or have no voice in the process. Another situation include professors are limited in the function of promoting academic integrity in many institutions.

\section{Conclusion}

Examination of institutional processes to promote academic integrity within online or virtual programs in higher education provided insight into the issue. Issues of academic integrity occur on local, national and international levels. Feedback from an international conference included comments from higher education faculty from Africa confirming the renewed concern regarding the rise in academic integrity. The planned behavior theory frames the 
study while the review of literature helps to ground this current research on academic integrity.

A culture of academic integrity can reduce negative or uncomfortable situations due to potential faculty judgement and adverse consequences for students. Promoting academic integrity through pedagogical processes can support the development of scholarship over punitive consequences [6]. A proactive approach to reminders of the fundamentals of academic integrity allows for intervals of instruction and reflection for scholars. Fostering a culture of academic integrity through an academic community beyond the course and within the institution can promote ethical responsibility.

\section{References}

[1] Bachore, M. (2016). The nature, causes and practices of academic dishonesty/cheating in higher education: The case of Hawassa University. Journal of Education and Practice,7(19). http://iiste.org/Journals/index.php/JEP/artic leview/31909/3 2779

[2] Bartlett, T. (2009). Cheating goes global as essay mills multiply. Chronicle of Higher Education, 55(28), pA1.

[3] Busch, P., \& Bilgin, A. (2014). Student and staff understanding and reaction: Academic integrity in an Australian university. Journal of Academic Ethics, 12(3), 227. doi: 10.1007/s10805-014-9214-2

[4] Cetin, B. (2015). Academic motivation and selfregulated learning in predicting academic achievement in college. Journal of International Education Research. 11(2), 95-106. http://files.eric.ed.gov/fulltext/EJ1060062.pdf

[5] Fähnrich, B., Danyi, C. J., \& Nothhaft, H. (2015). The german plagiarism crisis. Journal of Communication Management, 19(1), 20-38. doi.org/10/1108/JCOM1112013-0081

[6] Griffith, J. (2013). Pedagogical over punitive: The academic integrity websites of Ontario Universities. Canadian Journal of Higher Education, 43(1), 1-22. Retrieved from http://files.eric.ed.gov/fulltext/ EJ1007029.pdf

[7] Hart, H. (2014). Virtue in the virtual world: A model of academic integrity for online learning. University of Maryland University College. ProQuest Dissertations Publishing

[8] Ison, D. C. (2015). The influence of the internet on plagiarism among doctoral dissertations: An empirical study. Journal of Academic Ethics, 13(2), 151-166. doi:10.1007/s10805-015-7

[9] Jaggars, D. E. (2014). We can imagine the future, but are we equipped to create it? Portal: Libraries and the Academy, 14(3), 319-323. doi:10.1353/pla.2014.001

[10] Kivell, N.M., Evans, S.D. \& Paterson, S. E. (2017). Community power structural analysis and the ethical considerations of "studying up." American Journal of Community Psychology, 60, 467-475. doi:10.1002/ajcp.12190

[11] McGee, P. (2013). Supporting academic honesty in online courses. Journal of Educators Online, 10(1). http://files.eric.ed.gov/fulltext/EJ1004890.pdf

[12] Minarcik, J., \& Bridges, A. a. (2015). Psychology graduate students weigh in: Qualitative analysis of academic dishonesty and suggestion prevention strategies. Journal of Academic Ethics, 13(2), 1997-216. doi:10.1007/s10805- 105-9230-x.

[13] National Center for Education Statistics. (n.d.). Fast facts: Distance learning. Retrieved from https://nces.ed.gov/fastfacts/display.asp?id=80

[14] Soroya, M. S., Hashmi, M. A., \& Soroya, S. H. (2016). Academic integrity: Effects of demographic variables on students' conduct. South Asian Studies (1026-678X), 31(2), $27-43$.

[15] Stone, T.H., Jawahar, I.M., Kissamore, J. L. (2010). Predicting academic misconduct intentions and behavior using the theory of planned behavior and personality. Basic and Applied Social Psychology, 31(1), 35-45. doi:10.1080/01973530903539895

[16] Tolman, S. (2017). Academic dishonesty in online courses: Considerations for graduate preparatory programs in higher education. College Student Journal, 51(4), 579584. Retrieved from https://www.Thefree library .com/

[17] Quraishi, U., \& Aziz, F. (2017). Academic Dishonesty at the Higher Education Level in Punjab, Pakistan. Journal of Research \& Reflections In Education (JRRE), 11(1), 6682. Retrieved from http://ue.edu.pk/jrre/articles/ 1101007.pdf 